Marcel Fraix*, DO, MBA, Sondos Badran, BS, OMS II, Victoria Graham, PT, DPT, OCS, NCS, Donna Redman-Bentley, PT, PhD, Eric L. Hurwitz, DC, PhD, Valerie L. Quan, OD, Michelle Yim, DO, MPH, Mary Hudson-McKinney, PT, MS, DPT, NCS and Michael A. Seffinger, DO

\title{
Osteopathic manipulative treatment in individuals with vertigo and somatic dysfunction: a randomized, controlled, comparative feasibility study
}

https://doi.org/10.7556/jaoa.2020.147

Received April 22, 2019; accepted March 6, 2020; published online October 29, 2020

\section{Abstract}

Context: There is a paucity of research assessing the efficacy of osteopathic manipulative treatment (OMT) in patients with vertigo.

Objective: To assess the feasibility of conducting a randomized, controlled trial comparing OMT and vestibular rehabilitation therapy (VRT), alone or in combination, in patients with vertigo and somatic dysfunction.

Methods: Volunteers with vertigo who were also diagnosed with somatic dysfunction (SD) were prospectively enrolled in a blinded, randomized, controlled cohort comparative effectiveness study and assigned to 1 of 4 groups: OMT alone, VRT alone, a combination of OMT and VRT (OMT/ VRT), or a nonintervention control group. Participants between 18 and 79 years of age were included if they had experienced symptoms of vertigo for at least 3 months' duration, demonstrated somatic dysfunction, and could participate in computerized dynamic posturography (CDP)

*Corresponding author: Dr Marcel Fraix, DO, MBA, Western University of Health Sciences College of Osteopathic Medicine of the Pacific, Pomona, 309 E. Second St., Pomona, CA 91766-1854, USA, E-mail:mfraix@westernu.edu

Sondos Badran, BS, OMS II, Donna Redman-Bentley, PT, PhD, Valerie L. Quan, OD, Mary Hudson-McKinney, PT, MS, DPT, NCS and Michael A. Seffinger, DO, Western University of Health Sciences College of Osteopathic Medicine of the Pacific, Pomona, CA, USA Victoria Graham, PT, DPT, OCS, NCS, California State University Northridge, Northridge, USA

Eric L. Hurwitz, DC, PhD, The University of Hawaii, Manoa, USA Michelle Yim, DO, MPH, The Dignity Health California Hospital Medical Center, Los Angeles, CA, USA testing, tolerate manual therapy and exercises, and communicate effectively in English or Spanish. A total of 3 treatments lasting 45 minutes each were administered 1 week apart to each participant. OMT in this study consisted of counterstrain, myofascial release, balanced ligamentous tension, soft tissue, HVLA, and articulatory techniques. Comparisons were made between composite scores (CS) assessed with computerized dynamic posturography (CDP), dizziness handicap inventory (DHI), optometric evaluation, and osteopathic structural examinations collected before the first treatment, after the third/final treatment, and 3 months after the final treatment. (ClinicalTrials.gov number NCT01529151)

Results: A total of 23 patients were included in the study: 7 in the OMT group, 5 in the VRT group, 6 in the OMT/VRT group, and 5 in the control group. The OMT/VRT group demonstrated significant improvement in DHI score $(\mathrm{P}=0.0284)$ and CS $(\mathrm{P}=0.0475)$ between pre- and 3-month posttreatment measures. For total severity, improvements were significant in the OMT group both from pretreatment to immediate posttreatment measures $(\mathrm{P}=0.0114)$ and from pretreatment to 3-month posttreatment measures $(\mathrm{P}=0.0233)$. There was a statistical difference between the OMT and control groups from pretreatment to 3-month posttreatment DHI scores $(\mathrm{P}=0.0332)$. Also, there was a statistical difference in DHI score between VRT and control from pre- to 3-month posttreatment scores $(\mathrm{P}=0.0338)$. OMT/VRT statistically and clinically improved visual acuity in patients' right eyes from pre- to posttreatment $(\mathrm{P}=0.0325)$. In all participants, vergence dysfunction was prevalent $(5 ; 21.7 \%)$ in addition to vertical heterophoria (15; 65.2\%).

Conclusion: A combination of OMT and VRT significantly reduced vertigo and improved balance 3 months after treatment $(\mathrm{P}<0.05)$. There was a high prevalence in vergence and vertical heterophoria, which are not typical 
screening measurements used by physical therapists and physicians to assess vertigo patients. With a small sample size, this study demonstrated the feasibility of an interdisciplinary team evaluating and treating patients with vertigo in a community setting. A larger study is needed to assess the efficacy of OMT/VRT in vertigo patients.

Keywords: computerized dynamic posturography; osteopathic manipulative treatment; somatic dysfunction; vertigo; vestibular rehabilitation therapy.

Vertigo is defined by the American Academy of Otolaryngology - Head and Neck Surgery "as an illusory sensation of motion of either the self or the surroundings in the absence of true motion."1 It can be central and/or peripheral in origin. The less common centrally-mediated conditions include migrainous vertigo, brainstem ischemia, cerebellar infarction, and intracranial hemorrhage. Peripheral causes, which account for $80 \%$ of vertigo cases, include Meniere's disease, vestibular neuritis, and benign paroxysmal positional vertigo. ${ }^{2}$ Overall, vertigo is a relatively common problem in the general population, affecting approximately $15 \%$ to over $20 \%$ of adults yearly. ${ }^{3}$ It also has a significant impact on those it affects, both in terms of function and quality of life. ${ }^{4}$

Somatic dysfunction (SD), especially of the cervical spine, may be a cause of or contributing factor to vertigo. $\mathrm{SD}$ is defined as "impaired or altered function of related components of the somatic (body framework) system: skeletal, arthrodial, and myofascial structures, and related vascular, lymphatic, and neural elements." ${ }^{5}$ While the incidence of SD in those affected by vertigo is unknown, there are various hypotheses regarding potential pathogenic connections, one of which is that vertigo may arise from proprioceptive dysfunction in the cervical spine. Given the high density of mechanoreceptors in cervical zygapophyseal joints and soft tissues, there is evidence pointing to impaired proprioceptive and somatosensory function in patients experiencing cervical pain and vertigo. ${ }^{6-8}$ Patients suffering from whiplash injuries can experience persistent vertigo and have been noted to have impaired postural control. ${ }^{9-11}$ It has also been shown that degenerative disease of the cervical spine can result in impaired balance and cervical spondylosis may be a significant cause of vertigo in the elderly. ${ }^{12,13}$ Osteopathic manipulative treatment (OMT) may provide a means of treatment for those suffering from vertigo, particularly if SD plays a role.

Because of the potential role of proprioceptive and joint dysfunction in vertigo, manual approaches and vestibular rehabilitation are common treatments.
Manual approaches include chiropractic treatment, manual physical therapy, and OMT. The chiropractic literature suggests that spinal manipulation treatment (SMT) provides symptomatic relief in patients experiencing neck pain and vertigo. ${ }^{14,15}$ Two studies have demonstrated that OMT can be tolerated in patients with vertigo and may be useful in reducing imbalance and improving quality of life. ${ }^{16,17}$ Vestibular rehabilitation therapy (VRT) is recognized as a standard treatment for patients with vertigo. ${ }^{18}$ VRT aims to address impaired postural control and proprioception in those individuals affected by neck pain and vertigo. ${ }^{19-22}$ VRT uses a personalized approach for treatment by first categorizing the vestibular dysfunction in a structured examination. VRT is then directed to promote neuroplastic changes that normalize motor response to vestibular sensory input. ${ }^{23,24}$

The visual system can also contribute to symptoms of vertigo. $^{25-27}$ It provides spatial orientation and depth perception, and it detects visual motion. Vergence is the inward or outward turning of the eyes to maintain a single and binocular image of objects at varying distances. ${ }^{28,29}$ Vestibular ocular reflex (VOR) stabilizes gaze with head movement. VOR gain, or the amount of compensatory eye movement relative to the amount of head rotation, is modulated by viewing distance and vergence angle. Previous studies have suggested that deficits in vergence could affect gaze stabilization during body movement, causing double or blurry vision that could lead to vertigo and sensation of imbalance. ${ }^{30-32}$ Therefore, blurred vision and oculomotor dysfunctions can produce inappropriate VOR adjustment, which can be a cause of vertigo. ${ }^{33}$

The purpose of this study was to examine the effect of OMT in patients with a diagnosis of vertigo as well as the feasibility of conducting a randomized controlled trial comparing both OMT and VRT; to the best of our knowledge, there has been no published research examining both OMT and VRT in subjects suffering from vertigo that is peripheral in origin. We sought to assess how measures for vertigo, balance, and vision changed over time. Another aim of this study was to examine the relationship between $\mathrm{SD}$ in patients suffering from peripheral vertigo.

\section{Methods}

\section{Participants and research design}

Participants were recruited from the community surrounding the main campus of Western University of Health Sciences in Pomona, California. The Western University of 
Health Sciences institutional review board approved the procedures and interventions used in this study.

All prospective participants were required to complete a written screening questionnaire administered by a research assistant (S.B.) and were provisionally admitted to the study if they met the following inclusion criteria: age between 18 and 79 years; symptoms of vertigo for longer than 3 months' duration; ability to tolerate 30 minutes of sitting and standing; ability to transfer from sitting to standing and ambulate independently; ability to tolerate manual therapy and exercise; ability to understand instructions and communicate effectively in English or Spanish; and presence of SD. Patients were excluded if they had a medical history of acute traumatic injury, bleeding disorders, anticoagulation (coumadin) therapy, Meniere's disease, endolymphatic hydrops, neurological conditions (including peripheral neuropathy, stroke, traumatic brain injury, cerebral aneurysm, and multiple sclerosis), spinal trauma or history of cervical spine surgery, rheumatoid arthritis, legal blindness in 1 or both eyes, Ehlers-Danlos syndrome, or Down syndrome. Patients were also excluded if they refused to participate, if they were undergoing concurrent treatment with VRT, vision therapy, or manual approaches (OMT, chiropractic, etc.), or if they had received manual approaches within 3 months. The research assistant (S.B.) conducted an in person or telephone interview and reviewed the screening questionnaires to assess the eligibility of prospective participants and also obtained written informed consent from participants entered into the study. At each visit, participants received compensation in the form of $\$ 10$ gas cards to cover or reduce the cost of transporation. Prior to the start of the study, participants were recruited after being evaluated by an otolaryngologist in order to confirm a diagnosis of peripheral vertigo other than Meniere's disease and exclude vertigo of central origin.

This study was designed as a prospective, blinded, randomized, controlled cohort comparative effectiveness study in volunteer participants. (ClinicalTrials.gov No. NCT01529151) Block randomization was used to assign participants to 1 of 4 groups: OMT intervention alone, VRT intervention alone, OMT and VRT intervention together, or control. Participants admitted to the study were evaluated by an osteopathic physician who was board certified in NMM/ OMM, a Doctor of Physical Therapy (PT; M.H-M.), and a Doctor of Optometry (V.Q.). This evaluation included a history and physical examination, an osteopathic structural examination evaluating for SD, a physical therapy assessment to customize the active exercises per the VRT protocol, and an optometric evaluation assessing a series of visual processing functions. All evaluators (DO, PT [M.H-M.], and optometrist [V.Q.]) were blinded to the participants' group assignments. Additionally, the osteopathic physician who assessed participants for SD was not involved in the application of OMT. SD was recorded using the American Osteopathic Association (AOA)-approved subjective, objective, assessment, and plan (SOAP) note form for single organ system musculoskeletal examination. ${ }^{34}$ The osteopathic physician who provided OMT was not aware of the other osteopathic physician's assessment of SD and used his own assessment to guide the application of OMT; neither of these physicians is an author on the present study. Likewise, a PT (M.H-M) assessed each participant to categorize their vestibular dysfunction and a second PT (V.G.) provided the intervention. The researchers collecting outcomes measures remained blinded to group assignments, subject participation, and response to intervention. The osteopathic physician and PT (V.G.) providing the interventions were blinded to whether participants received 1 or both treatments.

\section{Outcomes measures}

Each participant was evaluated for SD before and after each intervention by the osteopathic physician who did not provide OMT. The OMT procedures used in this study included: counterstrain, myofascial release, balanced ligamentous tension, soft tissue, HVLA, and articulatory, as described in the Appendix, Table $\mathrm{A} 1^{35}$; these are described in further detail in a subsequent section of this article. A total severity score was calculated from 7 sections of the osteopathic SOAP note, which evaluate (1) the severity of tenderness, (2) asymmetry, (3) range of motion modified, and tissue texture changes (TART) in the (4) upper and lower cervical, (5) upper and lower thoracic, (6) lumbar, and (7) rib 1 and rib 2-5 regions. Each section was scored in a range from from 0 to 3 , with a higher score indicating more severity; 21 points was the maximum score that could be obtained for total severity.

All participants underwent repeat evaluation using the Sensory Organization Test (SOT) ${ }^{36}$ with Computerized Dynamic Posturography (CDP) ${ }^{37}$ and the Dizziness Handicap Inventory (DHI), ${ }^{36-38}$ osteopathic structural examination by the non-treating osteopathic physician (D.R.), and optometric evaluation. These measures were performed before treatment, postintervention at 3 weeks, and 3 months following the last treatment session to assess stability of the findings. Each treatment was 45 minutes long and treatments were spaced 1 week apart, so final measures were taken 3 months after the third and final treatment. Participants in the control groups also underwent repeat evaluation at the same 3 time points. The SOT composite score (CS) for balance and the DHI were the 


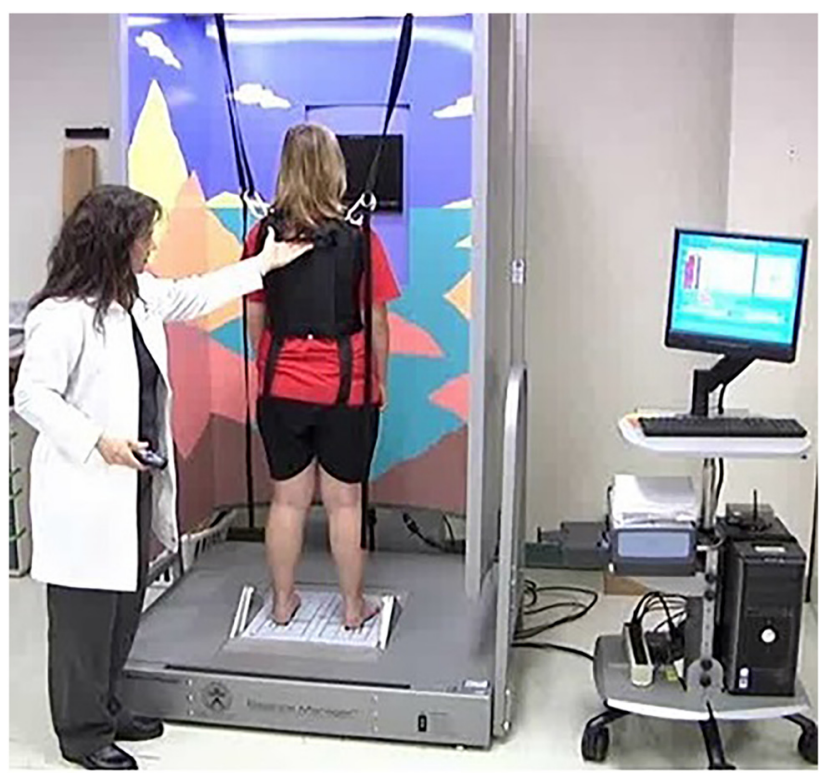

Figure 1: Sensory organization test with computerized dynamic posturography. Subjects' standing postural sway and reaction time is measured with force plates on the floor.

primary outcomes measures used to quantify a participant's degree of imbalance and vertigo, respectively.

The DHI is widely recognized as a validated symptom inventory and provides useful information regarding the evaluation and treatment of patients with vertigo. It consists of a 25 -item questionnaire with a maximum score of 100 points, indicating severe disability. The DHI has been shown to be reliable, valid, and sensitive to change, with 18 points indicating clinically important change. ${ }^{38-40}$ The SOT is also an accepted means by which to assess patients affected by balance disorders. Its use as an outcomes measure in patients who were treated with OMT for symptoms of vertigo has been previously evaluated. ${ }^{36}$ Subjects' standing postural sway and reaction time is measured with force plates on the floor (Figure 1). During an SOT evaluation, subjects stand with eyes open and closed for several minutes while the floor and/or walls moved around them, disadvantaging the visual, somatosensory, and vestibular systems. The maximum composite score (CS) is 100 points; higher points indicate less postural sway. Normative values are calculated based on age group to determine a cut-score separating balance response into discrete categories of either normal or poor (bottom $5^{\text {th }}$ percentile). An improvement of greater than 8 points is considered to be clinically significant. ${ }^{41} \mathrm{~A}$ research assistant (S.B.) in our study administered the CDP and was trained prior to doing so by a physical therapist (V.G.) who was participating in the study and had advanced training in CDP and VRT. This training included safe application of the overhead harness of the CDP machine as well as its operation and monitoring during testing.

Participants underwent a baseline (preintervention) vision evaluation by staff optometrists (V.Q.) from the NeuroOptometric Rehabilitation Service at the Eye Care Institute at Western University of Health Sciences. This evaluation was repeated at the completion of treatment and again at 3 months to assess the effect on vision function. Eight major categories were evaluated, including visual acuity, ocular alignment, fixation ability, pursuit and saccadic skills, depth perception, vergence skills, visual field status, and contrast sensitivity (Appendix, Table A2). All measures of the visual function were performed seated and without pharmacologic dilation of the pupil to limit temporary visual impairment or additional vertiginous side effects.

Monocular visual acuity was measured with Early Treatment Diabetic Retinopathy Study (ETDRS) letters, a method accepted by the National Eye Institute for accurate and repeatable visual acuity measurements across clinical studies involving vision..$^{42-44}$ Ocular alignment and vergence skills were analyzed utilizing the criteria in the Appendix, Table A2 to assign a diagnosis of convergence insufficiency (poor ability to turn eyes inward at a closer viewing angle) and vertical heterophoria (vertical misalignment of eyes) at baseline. Saccadic dysmetria was determined by observing horizontal eye movements for undershooting, overshooting, and ocular flutter using targets placed 45 degrees to the right and left.

\section{Treatment}

Patients in this study received OMT with the objective of treating diagnosed SD. This entailed the use of specific indirect and direct techniques, including soft tissue, inhibitory, myofascial release, articulatory, and highvelocity low-amplitude (HVLA) techniques. Muscle energy techniques, which are active and passive motion maneuvers similar to some VRT maneuvers, were not included as they could have confounded the data. Descriptions of the techniques used in this study are given in the Appendix, Table A1. Each participant in the OMT groups received a total of 3, 45-minute treatments, spaced approximately 1 week apart. The same physician performed all OMT techniques to ensure that they were applied as consistently as possible. Additionally, OMT was not restricted to a specific body region, since there is no evidence demonstrating that SD of a specific region causes or is correlated with vertigo. This is also in keeping with the 
theory of osteopathic medicine, which attempts to resolve structural imbalances and improve the overall function of the body.

Participants categorized as having peripheral vestibular hypofunction or motion hypersensitivity received condition-specific balance exercises in the sitting and standing positions that included gaze stabilization along with kinesthetic and proprioceptive retraining. A description of these techniques is listed in the Appendix, Table A3. Participants were given a monthly exercise $\log$ at the onset of treatment; compliance was self-reported and not tracked.

\section{Statistical analysis}

Our goal was to complete the study with a total of 80 participants. This number was determined based on DHI data from a pilot study. ${ }^{16}$ Eighty percent or greater power at the alpha $=0.05$ level (2-sided) is required to statistically detect clinically meaningful 18-point (or larger) between-group differences in DHI change scores. The number of participants in the study was based on the fact that in order to compute the specific power, either of the following would be needed: (1) number (or \%) of subjects in each group with anticipated clinically meaningful change; or (2) mean pre-

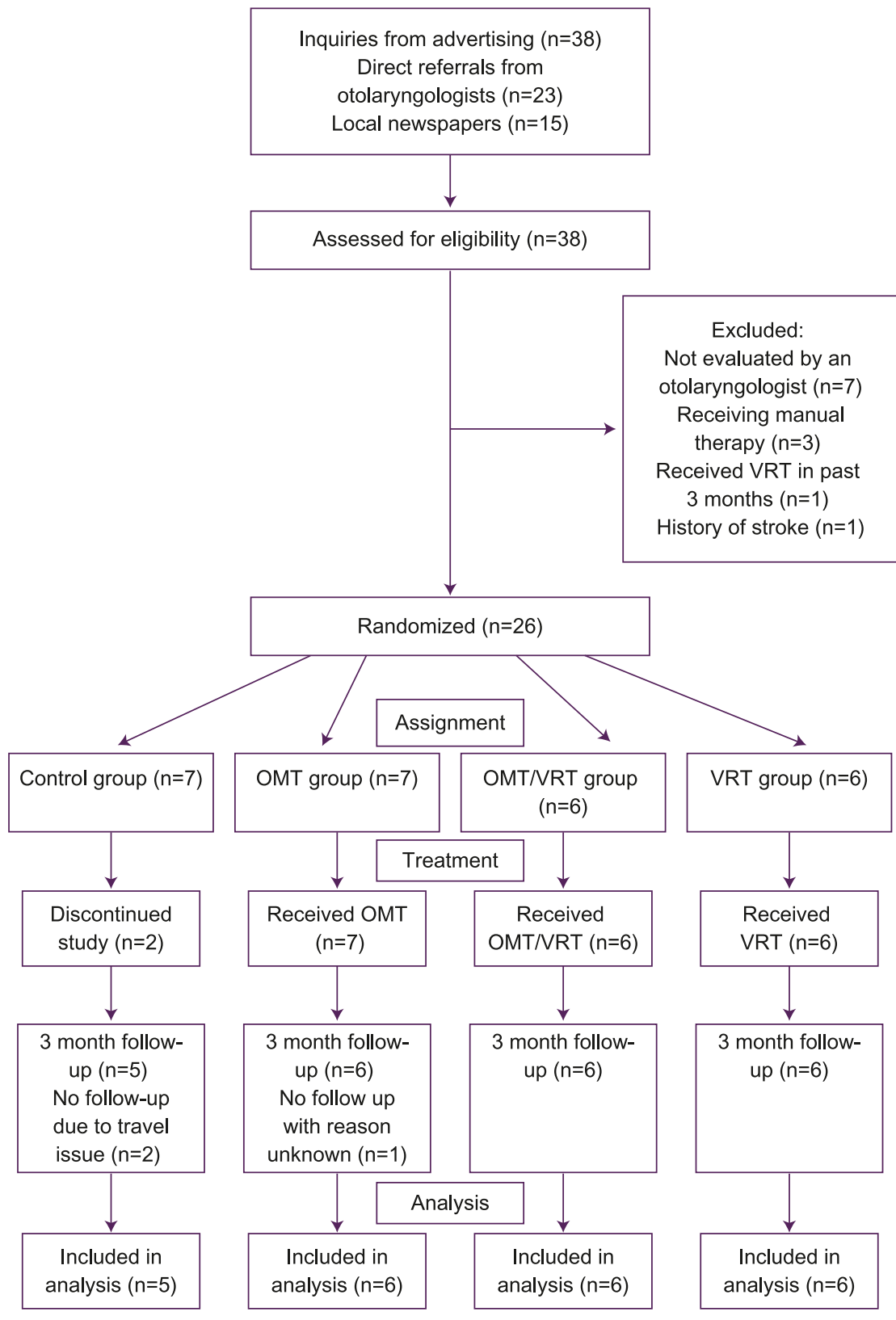

Figure 2: Flow chart demonstrating recruitment, inclusion and exclusion criteria, assignment, treatment, and analysis patterns for participants in this study. 
Table 1: Pretreatment, Posttreatment, and 3-Month Posttreatment Results (mean $n=23$ )

\begin{tabular}{|c|c|c|c|c|c|}
\hline Variable & Control $(n=5)$ & OMT $(n=7)$ & OMT/VRT $(n=6)$ & VRT $(n=5)$ & Total $(n=23)$ \\
\hline \multicolumn{6}{|c|}{$\begin{array}{l}\text { DHI Score } \\
\text { [mean (SD)], } \\
0-100 \text { Scale }\end{array}$} \\
\hline Pre & $33.2(29.2)$ & $48.3(20.7)$ & $36.3(19.2)$ & $45.2(20.5)$ & $41.2(21.7)$ \\
\hline Post & $37.2(34.8)$ & $41.7(24.0)$ & $28.0(16.4)$ & $37.6(28.4)$ & $36.3(24.8)$ \\
\hline 3 Month & $39.6(30.5)$ & $36.0(28.0)$ & $21.0(13.2)$ & $28.8(21.3)$ & $31.1(23.4)$ \\
\hline \multicolumn{6}{|c|}{$\begin{array}{l}\text { Composite Score } \\
\text { [mean (SD)], } \\
0-100 \text { scale }\end{array}$} \\
\hline Pre & $76.6(10.2)$ & $72.7(14.7)$ & $56.5(17.6)$ & $58.4(13.1)$ & $66.2(16.1)$ \\
\hline Post & $80.2(4.5)$ & $70.1(19.7)$ & $64.8(19.9)$ & $64.0(23.1)$ & $69.6(18.3)$ \\
\hline 3 Month & 79.4 (10.1) & $78.2(13.1)$ & $71.7(14.4)$ & $72.4(9.8)$ & $75.4(11.8)$ \\
\hline $\begin{array}{l}\text { Total Sev } \\
\text { Score [m } \\
0-21 \text { scal }\end{array}$ & & & & & \\
\hline Pre & $6.8(6.4)$ & $7.1(3.6)$ & 8.7 (3.3) & $4.4(1.5)$ & $6.9(4.0)$ \\
\hline Post & $4.0(2.9)$ & $3.1(2.7)$ & $7.3(5.7)$ & $7.0(4.9)$ & $5.2(4.3)$ \\
\hline 3 Month & $4.2(2.6)$ & $2.5(2.2)$ & $5.5(3.5)$ & $3.8(2.4)$ & $4.0(2.8)$ \\
\hline
\end{tabular}

post changes (and standard deviations) in DHI in each group or the between-group differences in means.

After completion of the treatment phase, descriptive statistics were used to characterize the study population at baseline, at the conclusion of treatment, and 3 months after treatment was concluded. Frequencies and percentages were computed for categorical variables, while means and standard deviations were computed for continuous variables. The differences between pretreatement and 3 weeks posttreatment results, pretreatment and 3 months posttreatment results, as well as 3 weeks posttreatment and 3 months posttreatment results included 95\% confidence intervals. Paired t-test and ordinary least-squares regression modeling were used to estimate and test effects of treatment on DHI, CS, total severity, and visual acuity within and between groups, respectively. Data management and statistical analyses were conducted with Microsoft Excel and SAS (version 9.1, Cary, NC).

\section{Results}

\section{Recruitment and compliance}

We assessed 38 subjects for eligibility, after which 26 subjects with vertigo of at least 3 months' duration were enrolled between January of 2012 and August 2014 to participate in this study. Twelve subjects were excluded: 7 had not been evaluated by an otolaryngologist, 3 were receiving concurrent treatment with manual therapy, 1 received VRT in the past 3 months, and 1 had a history of stroke. Of the 26 randomized participants, 7 (27\%) were assigned to the control group, 7 (27\%) to the OMT group, 6 (23\%) to the OMT/VRT group, and 6 (23\%) to the VRT group.

All subjects completed treatment in the OMT, VRT, and OMT/VRT groups. Two subjects in the control group did not complete follow-up evaluation at the end of week 3 nor at 3 months due to difficulty with travel to the clinic. One subject in the OMT group did not return for a 3-month posttreatment follow-up with reason unknown. Additionally, 1 subject in the VRT group did not complete the 3-week posttreatment or 3-month posttreatment follow-up. Therefore, data from a total of 23 subjects was used for analysis for pretreatment and 3-week posttreatment analysis; data from 23 subjects were used for 3-month posttreatment analysis. A flowchart showing progress of the participants throughout the study is available in Figure 2.

\section{Participant demographics}

The mean age and standard deviation of the 2 participants was $52.2 \pm 11.8$ years (range, 29-67 years). The majority (19; $73.1 \%)$ were women and $(7 ; 26.9 \%)$ were men. Ten $(38.5 \%)$ of the participants were employed full-time, 7 (26.9\%) part-time, and 9 (34.6\%) were not employed.

Additional characteristics noted at the time of visual testing included 13 participants (56.5\%) who wore 
Table 2: Within-Group Changes in DHI, CS, and Total Severity Scores

\begin{tabular}{|c|c|c|c|c|c|c|c|c|}
\hline \multirow[t]{2}{*}{ Variable } & \multicolumn{2}{|c|}{ Control $(n=5)$} & \multicolumn{2}{|c|}{ OMT $(n=7)$} & \multicolumn{2}{|c|}{ OMT/VRT $(n=6)$} & \multicolumn{2}{|c|}{ VRT $(n=5)$} \\
\hline & Change $(95 \% \mathrm{Cl})$ & P-value & Change $(95 \% \mathrm{Cl})$ & P-value & Change $(95 \% \mathrm{Cl})$ & P-value & Change $(95 \% \mathrm{Cl})$ & P-value \\
\hline \multicolumn{9}{|l|}{ DHI Score } \\
\hline Pre-Post & $-4.0(-14.4,6.4)$ & 0.3453 & $6.6(-8.0,21.1)$ & 0.3115 & $8.3(-10.7,27.4)$ & 0.3124 & $7.4(-9.0,23.8)$ & 0.2789 \\
\hline Pre- 3 Mo & $-6.4(-18.4,5.6)$ & 0.2116 & $10.3(-11.3,31.9)$ & 0.2740 & $15.3(2.4,28.2)$ & $0.0284^{\star}$ & $16.2(-4.4,36.8)$ & 0.0948 \\
\hline Post-3 Mo & $-2.4(-22.1,17.3)$ & 0.7527 & $1.67(-10.2,13.5)$ & 0.7320 & $7.0(-1.3,15.3)$ & 0.0814 & $8.8(-14.6,32.2)$ & 0.3554 \\
\hline \multicolumn{9}{|l|}{ Composite } \\
\hline \multicolumn{9}{|l|}{ Score } \\
\hline Pre-Post & $-3.6(-13.7,6.5)$ & 0.3788 & $2.6(-3.8,8.9)$ & 0.3591 & $-8.3(-17.7,1.1)$ & & $-5.6(-29.0,17.8)$ & 0.5428 \\
\hline Pre- 3 Mo & $-2.8(-11.9,6.3)$ & 0.4430 & $-0.7(-9.4,8.0)$ & 0.8520 & $-15.2(-30.1,-0.2)$ & $0.0475^{\star}$ & $-14.0(-30.8,2.8)$ & 0.0814 \\
\hline Post-3 Mo & $0.8(-8.9,10.5)$ & 0.8310 & $-2.2(-7.2,2.9)$ & 0.3223 & $-6.8(-21.1,7.4)$ & 0.2745 & $-8.4(-27.7,10.9)$ & 0.2932 \\
\hline \multicolumn{9}{|c|}{ Total Severity } \\
\hline Pre-Post & $2.8(-4.2,9.8)$ & 0.3285 & $4.0(1.3,6.7)$ & $0.0114^{\star}$ & $1.3(-5.7,8.3)$ & 0.6457 & $-1.6(-6.6,3.4)$ & 0.4256 \\
\hline Pre- 3 Mo & $2.6(-5.5,10.7)$ & 0.4219 & $5.0(1.0,9.0)$ & $0.0233^{*}$ & $3.2(-2.4,8.7)$ & 0.2010 & $0.6(-1.8,3.0)$ & 0.5291 \\
\hline Post-3 Mo & $-0.2(-3.6,3.2)$ & 0.8798 & $0.8(-1.0,2.6)$ & 0.2892 & $1.8(-2.4,6.0)$ & 0.3148 & $2.2(-3.3,7.7)$ & 0.3299 \\
\hline
\end{tabular}

${ }^{*} p<0.05$ for within-group analysis for each treatment and control group. Abbreviations: CS, composite scores; DHI, dizziness handicap inventory (DHI)

multifocal spectacle lenses during the study, $6(26.1 \%)$ who had defective or poor stereopsis at baseline, and $6(28.6 \%)$ who had visual field deficits. Of the 23 participants, 21 (91.3\%) reliably completed visual field testing.

\section{Clinical outcomes}

Mean and standard deviation for DHI, composite, and total severity scores for pretreatment, 3-week posttreatment, and 3-month posttreatment evaluations were calculated (Table 1). Participants in the OMT, OMT/VRT, and VRT groups exhibited improvement in DHI scores over time. Participants saw an improvement of mean DHI scores from 48.3 to 36.0 in the OMT group, from 36.3 to 21.0 in the in the OMT/VRT group, and from 45.2 to 28.8 in the the VRT group. Only mean DHI scores in the control group showed an increase, from 33.2 to 39.6. Participants in the OMT/VRT and VRT groups improved their balance composite scores (CS) over time. The OMT/VRT group had an increase from 56.5 to 71.7 on composite score mean and the VRT group had a mean increase from 58.4 to 72.4 on mean composite score. Smaller mean composite score improvements were seen in the OMT group, from 72.7 to 78.2 , and in the control group, from 76.6 to 79.4. Total severity score on the osteopathic evaluation decreased over time in the OMT/VRT group from 8.7 to 5.5, in the OMT group from 7.1 to 2.5 , then more minimally in the VRT group, from 4.4 to 3.8 , and in the control group from 6.8 to 4.2 .

Paired sample t-tests were conducted to determine statistical significance between pretreatment, 3-week posttreatment, and 3-month posttreatment values (Table 2). The OMT/VRT group significantly improved in DHI score with a 15.3 (95\% confidence interval [CI], 2.4-28.2; $\mathrm{P}=0.0284$ ) decrease in mean and improved CS of 15.2 between pretreatment and 3-month posttreatment values (95\% CI, 3.1-..2; $\mathrm{P}=0.0475$ ). For total severity, the OMT group significantly improved from pretreatment to posttreatment by 4 points ( $95 \% \mathrm{CI}, 1.3-6.7 ; \mathrm{P}=0.0114)$ and from pretreatment to 3-month posttreatment values by 5 points (95\% CI, 1.0-9.0; $\mathrm{P}=0.0233$ ).

Between-group differences were analyzed using ordinary least-squares regression modeling (Table 2). There was a statistical difference in DHI score between the OMT/VRT and control groups from pretreatment to 3-month posttreatment measures $(\mathrm{P}=0.0332)$. Also, there was a statistical difference in DHI score between the VRT and control groups from pretreatment to 3-month posttreatment results $(\mathrm{P}=0.0338)$. There were no statistically significant changes in CS or total severity score between groups over time.

OMT, OMT/VRT, and VRT results exhibited clinical significance in DHI and CS (Table 3). Although no participants in the control group exhibited clinical significance in DHI score, a participant in the control group $(1 ; 20 \%)$ demonstrated clinical significance in CS. Table 3 illustrates the percentage of participants in each group who showed improvement in results when comparing pretreatment vs 3-week posttreatment measures, pretreatment vs 3-month posttreatment measures, pretreatment vs 3-month posttreatment measures, and 3-week posttreatment vs 3-month posttreatment measures. The OMT/VRT group demonstrated improvement in DHI from pretreatment to 3-month posttreatment $(3 ; 50 \%)$, in CS at pretreatment vs 
Table 3: Clinically Significant* Changes Over Time, $n(\%)$

\begin{tabular}{lrrrr}
\hline Variable & $\begin{array}{r}\text { Control } \\
(\mathbf{n}=5)\end{array}$ & $\begin{array}{r}\text { OMT } \\
(\mathbf{n}=7)\end{array}$ & $\begin{array}{r}\text { OMT/VRT } \\
(\mathbf{n}=6)\end{array}$ & $\begin{array}{r}\text { VRT } \\
(\mathbf{n}=5)\end{array}$ \\
\hline $\begin{array}{l}\text { DHI Score } \\
\text { Pre-Post }\end{array}$ & $0(0.0)$ & $1(14.3)$ & $1(16.7)$ & $2(40.0)$ \\
$\begin{array}{l}\text { Pre-3 Month } \\
\text { Post-3 }\end{array}$ & $0(0.0)$ & $2(33.3)$ & $3(50.0)$ & $2(40.0)$ \\
Month & $0(0.0)$ & $2(33.3)$ & $1(16.7)$ & $1(20.0)$ \\
Composite Score & & & & \\
Pre-Post & $1(20.0)$ & $0(0.0)$ & $3(50.0)$ & $2(40.0)$ \\
Pre-3 Month & $1(20.0)$ & $1(16.7)$ & $4(66.7)$ & $4(80.0)$ \\
Post-3 & $0(0.0)$ & $1(16.7)$ & $2(33.3)$ & $2(40.0)$ \\
Month & & & & \\
\hline
\end{tabular}

posttreatment $(3 ; 50 \%)$, and pretreatment vs 3 -month posttreatment $(4 ; 66.7 \%)$. The VRT group improved in CS at pretreatment-3- month posttreatment (4; 80\%). All intervention groups showed some percentage of participants improving over all periods of time except the OMT group in CS from pretreatment to posttreatment.

Percentage of participants that had somatic dysfunctions in the different treatment groups were evaluated. Compared to the control group, most somatic dysfunctions did not change significantly in terms of the total severity score. Percentage of participants with postural abnormality decreased by $100 \%$ with all 7 paticipants in the OMT group, $50 \%$ or 3 patricpants in the OMT/VRT group, and $100 \%$ or all 5 participants in the VRT groups from pretreatment to 3 months posttreatment. However, the 6 participants in the control group remained constant $(0 \%$ change in percentage of partipcants with postural abnormality).

Ocular dysfunctions were also evaluated. There was a higher frequency of oculomotor dysfunctions and improvement of 18 points on the DHI and more than 8 points on the Composite Score represent clinically significant changes. Abbreviations: DHI, dizziness handicap inventory (DHI) poor binocular fusion compared to a normal population. Vergence dysfunction, particularly convergence insufficiency, was more prevalent in all the groups (5; $21.7 \%)$ compared to normal $(2-5 \%)$ and remained the same pretreatment and posttreatment. ${ }^{45}$ Vertical heterophoria, which is the tendency of 1 eye to drift upward when fusion between 2 eyes is disrupted, was also more prevalent in all the groups $(15 ; 65.2 \%)$ compared to normal $(10-20 \%)^{46}$ Dysmetric eye movement improved over time only in the OMT/VRT group. From pretreatment to 3 months posttreatment, the percentage change of dymestria was $50 \%$, with the prevalence of dysmetria decreasing from $66.7 \%$ (4) to $33.3 \%$ (2). A statistical improvement ( $\mathrm{n}=6$; $\mathrm{P}=0.0325$ ) in monocular visual acuity in the right eye was

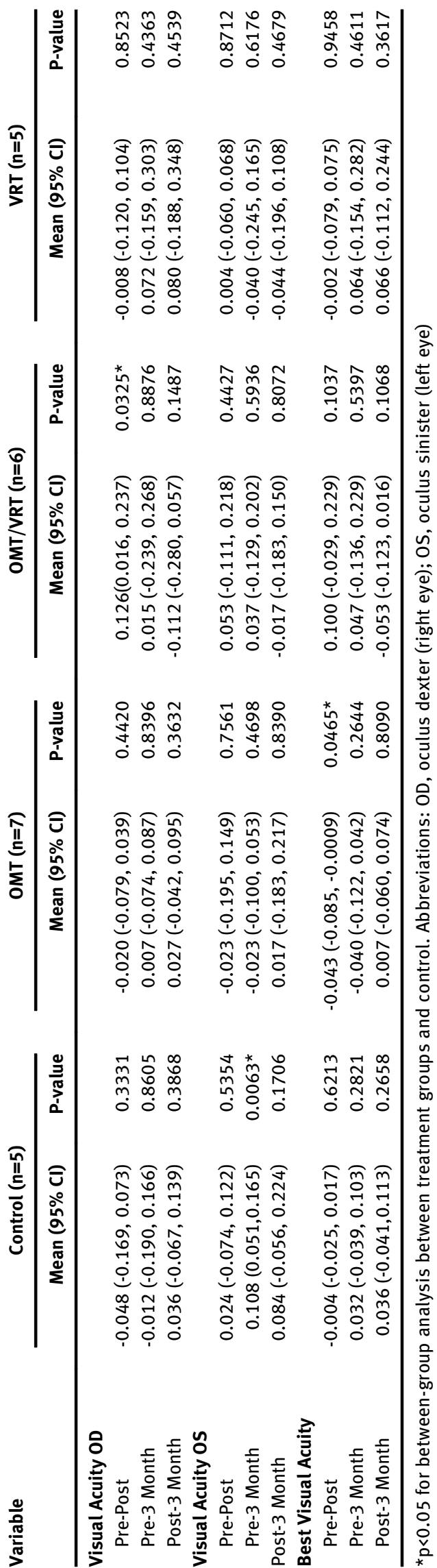


seen in the OMT/VRT group with a logMar change of 0.126 (95\% CI, 0.016-0.237) (Table 4). A similar, but smaller change, was seen in the control group's left eye visual acuity from pretreatment to 3-month posttreatment with a logMar change of 0.108 ( $95 \%$ CI, 0.051-0.165; $\mathrm{P}=0.0063$ ). The Snellen equivalent was a 1-line improvement from 20/20 line to 20/15 in both groups, with the greatest change in the OMT/VRT group. Between-group differences in visual acuity were conducted (data not shown). There was a statistical difference between OMT/VRT and control for visual acuity in the right eye (0.0044; $95 \% \mathrm{CI}, 0.085-0.0009)$ and best visual acuity from the right or left eye $(\mathrm{P}=0.0321)$ from pretreatment to posttreatment.

\section{Adverse events}

One subject reported harm, which was due to a fall while exiting the overhead harness of the Computerized Dynamic Posturography (CDP) machine. The subject had completed testing. This was reported to the IRB Committee at Western University of Health Sciences, and other than self- limited soreness and bruising which resolved spontaneously in a few days, no serious injury was sustained.

\section{Discussion}

Based on the results, participants that were in the OMT/VRT group statistically improved their balance composite score and reduced their DHI score, indicating that the participants were more balanced and experienced less vertigo. Although the effects were not immediate, after 3 months of treatment, the participants had improved their scores compared to before treatment. Furthermore, group analysis demonstrated that there was a statistical significance between OMT/VRT and control group from pre to 3-month posttreatment for DHI score. Improving a participant's vertigo subjectively and objectively is important clinically because it leads to an enhanced quality of life. Therefore, a combination of a standard treatment for vertigo patients, VRT, as well as OMT seems to have the most statistically significant improvement. The results should be interpreted with caution due to the small sample size in this study.

The vision data identified a high prevalence of vergence disorder and vertical hetereophoria, which others have shown to confound vestibular gain and postural stability. ${ }^{47,48}$ Change or improvement in these areas from pre to post was not seen nor expected as there was no active vision intervention in this study. It is a logical next step to investigate the effect of corrective interventions, which include vergence training and spectacle prism, on balance. It is also important to mention that visual acuity, pursuit, and saccades are typical vision measures captured during a balance evaluation. This study utilized and proposes an expanded vision evaluation adding vergence dysfunction and vertical heterophoria, which were found at a higher prevalence in our subjects. The effect on dysmetria and visual acuity, which are commonly measured, were both found to improve in the combined OMT/VRT group possibly due to improved gaze stabilization and coordination of visual-vestibular interaction. Some improvement in visual acuity was also seen within the control group. However, the change was less than 5 letter difference $(\log M a r= \pm 0.11) .{ }^{49}$ This suggests that the results do not reflect a true change in visual acuity and may be attributed to test-retest variability. The visual acuity change in the OMT/VRT group met the threshold to reflect true change and could be a result of rehabilitation. Visual acuity improvement was reliably detected in the OMT/VRT group after rehabilitation. A larger sample size for all groups would be needed to compare the preliminary trends seen in this study.

The evaluation and treatment of participants was successfully coordinated amongst three types of blinded providers, including Doctors of Osteopathic Medicine, Physical Therapy, and Optometry. The protocol design presented few to no obstacles in terms of flow of participants through the study. Having a centralized site was helpful in facilitating interdisciplinary coordination and participants had little difficulty in terms of scheduling and the actual location and layout of the clinic. Additionally, evaluation and treatment of subjects was well tolerated and only one minor adverse event was experienced by a patient who fell after the overhead harness of the CDP machine was removed.

Despite obtaining statistically significant results, the results should be taken with caution due to the small sample size, which was the major limitation of the study. The most significant factors contributing to a small sample size were lack of funding for advertisement and participant compensation and travel-related issues. Future studies must take these factors into consideration in order to recruit and enroll an adequate number of participants.

There is still limited research regarding the application of manual therapy in patients suffering from vertigo, especially with regards to OMT. This study is valuable since 
it demonstrates that the study protocol and interdisciplinary coordination employed are feasible. The study presents a novel interdisciplinary approach and comparison of OMT and VRT, both alone and in combination. To the best of our knowledge it is also the first study that looks at the relationship between somatic dysfunction and vertigo as well as separates the evaluation of somatic dysfunction from its treatment with OMT.

\section{Conclusion}

This feasibility and interdisciplinary study revealed clinical significant results in the OMT, VRT, and OMT/VRT treatment groups. OMT, in combination with VRT, may be helpful in treating patients with vertigo patients. Further research is needed to validate the results and investigate the role of OMT/VRT in improving vertigo, balance composite score, and visual acuity.

Acknowledgements: We would like to thank David Redding, DO and Raymond Hruby, DO, MS, FAAO for performing the osteopathic evaluation and treatment of study participants.

Support: This study was funded by the American Osteopathic Association and the Osteopathic Heritage Foundation; funds supported expenses related to reimbursement for study participant travel, research assistant salary, consultant services for study design and statistical analysis, and calibration of equipment.

Author contributions: All authors provided substantial contributions to conception and design, acquisition of data, or analysis and interpretation of data; all authors drafted the article or revised it critically for important intellectual content; all authors gave final approval of the version of the article to be published; and all authors agree to be accountable for all aspects of the work in ensuring that questions related to the accuracy or integrity of any part of the work are appropriately investigated and resolved.

Informed consent: All participants provided written informed consent for this study.

Ethical approval: The Institutional Review Board at Western University of Health Sciences approved this study. This study was prospectively registered at ClinicalTrials.gov (no. NCT01529151).

Financial disclosure: None reported.

Disclaimer: Dr Seffinger, an Associate Editor of the JAOA, was not involved in the editorial review or decision to publish this article.

\section{References}

1. Bhattacharyya N, Gubbels SP, Schwartz SR, et al. Clinical Practice Guideline: Benign Paroxysmal Positional Vertigo (Update). Otolaryngol Head Neck Surg. 2017;156(3_suppl): S1-S47. doi: 10.1177/0194599816689667

2. Lai YT, Wang TC, Chuang LJ, Chen MH, Wang PC. Epidemiology of vertigo: a national survey. Otolaryngol Head Neck Surg. 2011 Jul; 145 (1):110-6. doi: 10.177/0194599811400007

3. Neuhauser HK. The epidemiology of dizziness and vertigo. Handb Clin Neurol. 2016;137:67-82. doi: 10.1016/B978-0-444-63437-5.00005-4

4. Lopez-Escamez JA, Gamiz MJ, Fernandez-Perez A, Gomez-Fiñana M, Sanchez-Canet I. Impact of treatment on health-related quality of life in patients with posterior canal benign paroxysmal positional vertigo. Otol Neurotol. 2003;24(4):637-641. doi: 10.1097/ 00129492-200307000-00018

5. Glossary Review Committee, for the Educational Council on Osteopathic Principles and the American Association of Colleges of Osteopathic Medicine. Glossary of Osteopathic Terminology. April 2002.

6. Li Y, Peng B. Pathogenesis, diagnosis, and treatment of cervical vertigo. Pain Physician. 2015;18(4):E583-E595.

7. Magnusson M, Malmström EM. The conundrum of cervicogenic dizziness. Handb Clin Neurol. 2016;137:365-369. doi: 10.1016/ B978-0-444-63437-5.00026-1

8. Yacovino DA, Hain TC. Clinical characteristics of cervicogenicrelated dizziness and vertigo. Semin Neurol. 2013;33(3):244-255. doi: $10.1055 / \mathrm{s}-0033-1354592$

9. Rubin AM, Woolley SM, Dailey VM, Goebel JA. Postural stability following mild head or whiplash injuries. Am J Otol. 1995;16(2):216-221.

10. Endo K, Suzuki H, Yamamoto K. Consciously postural sway and cervical vertigo after whiplash injury. Spine (Phila Pa 1976). 2008;33 (16):E539-E542. doi: 10.1097/BRS.0b013e31817c55fe

11. Treleaven J, Jull G, Sterling M. Dizziness and unsteadiness following whiplash injury: characteristic features and relationship with cervical joint position error. J Rehabil Med. 2003;35(1):36-43. doi: 10.1080/16501970306109

12. Freppel S, Bisdorff A, Colnat-Coulbois S, et al. Visuoproprioceptive interactions in degenerative cervical spine diseases requiring surgery. Neuroscience. 2013;255:226-232. doi: 10.1016/j.neuroscience.2013.09.060

13. Colledge NR, Barr-Hamilton RM, Lewis SJ, Sellar RJ, Wilson JA. Evaluation of investigations to diagnose the cause of dizziness in elderly people: a community based controlled study. BMJ. 1996; 313 (7060):788-792. doi: 10.1136/bmj.313.7060.788

14. Humphreys BK, Peterson C. Comparison of outcomes in neck pain patients with and without dizziness undergoing chiropractic treatment: a prospective cohort study with 6 month follow-up. Chiropr Man Therap. 2013;21(1):3. doi: 10.1186/2045-709X-21-3

15. Strunk RG, Hawk C. Effects of chiropractic care on dizziness, neck pain, and balance: a single-group, preexperimental, feasibility study. J Chiropr Med. 2009;8(4):156-164. doi: 10.1016/j.jcm. 2009.08.002

16. Fraix M. Osteopathic manipulative treatment and vertigo: a pilot study. PM R. 2010;2(7):612-618. doi: 10.1016/j.pmrj.2010.04.001

17. Papa L, Amodio A, Biffi F, Mandara A. Impact of osteopathic therapy on proprioceptive balance and quality of life in patients with dizziness. J Bodyw Mov Ther. 2017;21(4):866-872. doi: 10.1016/j.jbmt.2017.03.001 
18. Cabrera Kang CM, Tusa RJ. Vestibular rehabilitation: rationale and indications. Semin Neurol. 2013;33(3):276-285. doi: $10.1055 / \mathrm{s}-0033-1354593$

19. Grod JP, Diakow PR. Effect of neck pain on verticality perception: a cohort study. Arch Phys Med Rehabil. 2002;83(3):412-415. doi: 10.1053/apmr.2002.29660

20. Yahia A, Ghroubi S, Jribi S, et al. Chronic neck pain and vertigo: is a true balance disorder present?. Ann Phys Rehabil Med. 2009;52 (7-8):556-567. doi: 10.1016/j.rehab.2009.07.033

21. Stanton TR, Leake HB, Chalmers KJ, Moseley GL. Evidence of impaired proprioception in chronic, idiopathic neck pain: systematic review and meta-analysis. Phys Ther. 2016;96(6):876-887. doi: 10.2522/ptj. 20150241

22. de Vries J, Ischebeck BK, Voogt LP, et al. Joint position sense error in people with neck pain: a systematic review. Man Ther. 2015;20 (6):736-744. doi: 10.1016/j.math.2015.04.015

23. Telian SA, Shepard NT. Update on vestibular rehabilitation therapy. Otolaryngol Clin North Am. 1996;29(2):359-371.

24. Whitney SL, Sparto PJ. Principles ofvestibular physical therapy rehabilitation. Neurorehabilitation. 2011;29(2):157-166. doi: 10.3233/nre-2011-0690

25. Nashner LM. Organization and programming of motor activity during posture control. Prog Brain Res. 1979;50:177-184. doi: 10.1016/S0079-6123(08)60818-3

26. Nashner L, Shupert CL, Horak F, Black FO. Organization of postural control: an analysis of sensory and mechanical constraints. Progress in Brain Research. 1989;80:411-8; discussion 395-7.

27. Brandt T. Vertigo: Its Multisensory Syndromes, 2nd ed. Berlin: Springer; 1999.

28. Leigh RJ, Zee D. The Neurology of Eye Movements, 4th ed. New York: Oxford University Press; New York; 2006.

29. Ciuffreda KJ, Tannen B. Eye Movement Basics for the Clinician. St. Louis: Mosby Year Book, 1995.

30. Anoh-Tanon MJ, Bremond-Gignac D, Wiener-Vacher SR. Vertigo is an underestimated symptom of ocular disorders: dizzy children do not always need MRI. Pediatr Neurol. 2000;23(1):49-53. doi: 10.1016/s0887-8994(00)00140-5

31. Bucci MP, Lê TT, Wiener-Vacher S, Brémond-Gignac D, Bouet A, Kapoula Z. Poor postural stability in children with vertigo and vergence abnormalities. Invest Ophthalmol Vis Sci. 2009;50(10): 4678-4684. doi: 10.1167/iovs.09-3537

32. Winkler PA, Ciuffreda KJ. Ocular fixation, vestibular dysfunction, and visual motion hypersensitivity. Optometry. 2009;80(9):502-512. doi: 10.1016/j.optm.2009.01.014

33. Anand V, Buckley JG, Scally A, Elliott DB. Postural stability in the elderly during sensory perturbations and dual tasking: the influence of refractive blur. Invest Ophthalmol Vis Sci. 2003; 44(7):2885-2891. doi: 10.1167/iovs.02-1031

34. Ehrenfeucter WC. Chapter 34. Screening Osteopathic Structural Examination and Ch. 35 Segmental Motion
Testing. In: Chila AG, executive ed. Foundations of Osteopathic Medicine. 3rd ed. Baltimore, MD: Lippincott Williams \& Wilkins; 2011:410-436.

35. American Osteopathic Association. Foundations of Osteopathic Medicine. 3rd ed. Chila A, ed. Philadelphia, PA: Lippincott Williams \& Wilkins.

36. Fraix M, Gordon A, Graham V, Hurwitz E, Seffinger MA. Use of the SMART Balance Master to quantify the effects of osteopathic manipulative treatment in patients with dizziness. J Am Osteopath Assoc. 2013;113(5):394-403.

37. Allum JH, Shepard NT. An overview of the clinical use of dynamic posturography in the differential diagnosis of balance disorders. J Vestib Res. 1999;9(4):223-252.

38. Mutlu B, Serbetcioglu B. Discussion of the dizziness handicap inventory. J Vestib Res. 2013;23(6):271-277. doi: 10.3233/VES-130488

39. Jacobson GP, Newman CW. The development of the Dizziness Handicap Inventory. Arch Otolaryngol Head Neck Surg. 1990;116 (4):424-427. doi: 10.1001/archotol.1990.01870040046011

40. Clendaniel RA. Outcome measures for assessment of treatment of the dizzy and balance disorder patient. Otolaryngol Clin North Am. 2000;33(3):519-533. doi: 10.1016/s0030-6665(05)70225-5

41. Wrisley DM, Stephens MJ, Mosley S, Wojnowski A, Duffy J, Burkard R. Learning effects of repetitive administrations of the sensory organization test in healthy young adults. Arch Phys Med Rehabil. 2007;88(8):1049-1054. doi: 10.1016/j.apmr.2007.05.003

42. Kniestedt C, Stamper RL. Visual acuity and its measurement. Ophthalmol Clin North Am. 2003;16(2):155-v. doi: 10.1016/ s0896-1549(03)00013-0

43. Ricci F, Cedrone C, Cerulli L. Standardized measurement of visual acuity. Ophthalmic Epidemiol. 1998;5(1):41-53. doi: 10.1076/ opep.5.1.41.1499

44. Varma R, McKean-Cowdin R, Vitale S, Slotkin J, Hays RD. Vision assessment using the NIH Toolbox [published correction appears in Neurology. 2013;80(24):2277]. Neurology. 2013;80(11 Suppl 3): S37-S40. doi: 10.1212/WNL.0b013e3182876e0a

45. Cooper J, Duckman R. Convergence insufficiency: incidence, diagnosis, and treatment. J Am Optom Assoc. 1978;49:673-80.

46. Griffin JR, Borsting EJ. Binocular Anomalies, 5th ed. Optometric Extension Program Foundation; California; 2010.

47. Kapoula Z, Gaertner C, Yang Q, Denise P, Toupet M. Vergence and standing balance in subjects with idiopathic bilateral loss of vestibular function. PLoS One. 2013;8(6):e66652. doi: 10.1371/ journal.pone.0066652

48. Jackson DN, Bedell HE. Vertical heterophoria and susceptibility to visually induced motion sickness. Strabismus. 2012;20(1): 17-23. doi: 10.3109/09273972.2011.650813

49. Rosser DA, Cousens SN, Murdoch IE, Fitzke FW, Laidlaw DA. How sensitive to clinical change are ETDRS logMAR visual acuity measurements? Invest Ophthalmol Vis Sci. 2003;44(8): 3278-3281. doi: 10.1167 /iovs.02-1100 


\section{Appendix}

Table A1: OMT Techniques

\begin{tabular}{ll}
\hline Technique & Description \\
\hline Counterstrain & $\begin{array}{l}\text { Somatic dysfunction is considered to be due to continuing inappropriate strain reflex, which is inhibited by } \\
\text { applying a position of mild strain in the direction exactly opposite to that of the reflex; this is accomplished by } \\
\text { specific directed positioning about the point of tenderness to achieve the desired therapeutic response. } \\
\text { With continual palpatory feedback, the myofascial tissues are engaged by guiding them in the direction of least } \\
\text { resistance until tissue release occurs and increased movement is achieved. } \\
\text { Myofascial Release }\end{array}$ \\
$\begin{array}{l}\text { The ligaments and joints are placed in a position that facilitates release of tension and increased motion. } \\
\text { Tension }\end{array}$ & $\begin{array}{l}\text { A technique that usually involves lateral stretching, deep pressure, traction and/or separation of muscle origin } \\
\text { and insertion while monitoring tissue response and motion changes by palpation. } \\
\text { An osteopathic technique employing a rapid, therapeutic force of brief duration that travels a short distance within } \\
\text { the anatomic range of motion of a joint, and that engages the restrictive barrier in one or more planes of motion to } \\
\text { elicit release of restriction. } \\
\text { A low velocity/moderate to high amplitude technique in which a joint is carried through its full range of motion with } \\
\text { the therapeutic goal of increased range of motion. The activating force is either a repetitive springing motion or } \\
\text { repetitive concentric movement of the joint though the restrictive barrier. }\end{array}$ \\
\hline
\end{tabular}

Table A2: Neuro-Optometric Assessment

\begin{tabular}{lll}
\hline Area evaluated & Technique used & Normal criteria for vision tests \\
\hline Visual acuity & Precision Vision charts with ETDRS letters. & Monocular visual acuity $\leq 0.1$ \\
Ocular alignment & Unilateral and alternating cover test assessed alignment at 20 feet and & Distance: $<4$ exophoria or $<2$ esophoria \\
& $40 \mathrm{~cm}$ with prism bar neutralization. Saladin card was also used to & Near: $<7$ exo or $<1$ eso \\
& measure fixation disparity and phoria. & Vertical: no heterophoria \\
Fixation ability & Gaze holding was assessed monocularly and binocularly for 10 secs at & Absence of nystagmus or saccadic \\
& $40 \mathrm{~cm}$. & intrusions \\
Pursuit and saccade & Versional and saccadic eye movements were observed using fixation & Absence of restrictions, latency, lag or \\
ability & wands. & dysmetria. \\
Depth perception & Randot tests, requiring participants to wear polarized filters and identify & Local stereopsis $<50$ seconds. \\
& six geometric forms from random dot backgrounds, ranging from 500 to & \\
Vergence skills & 20 seconds of arc to measure stereopsis. & Distance NFV: $>3$ break $>1$ recovery \\
& Vergence ranges at 20 feet and $40 \mathrm{~cm}$ was measured with prism bars. & Distance PFV: $>3$ break $>4$ recovery \\
& & Near NFV: $>6$ break $>4$ recovery \\
& & Near PFV: $>9$ break $>6$ recovery. \\
Visual field status & Nova Vision head mounted perimeter assessed the extent of peripheral & 122 points seen is expected \\
& vision. & \\
Contrast sensitivity & Mars Letter Contrast Sensitivity Test was conducted monocularly. & $1.48-1.51$ log units ( $\leq 60$ yrs old). \\
& & $1.52-1.76$ log units $(>60$ yrs old)
\end{tabular}

Abbreviations: ETDRS, early treatment diabetic retinopathy study; NFV, negative fusional vergence; PFV, positive fusional vergence 
Table A3: Vestibular Rehabilitation Techniques

\begin{tabular}{|c|c|c|}
\hline $\begin{array}{l}\text { Vestibular dysfunction } \\
\text { category }\end{array}$ & Clinical confirming tests & Vestibular rehabilitation technique \\
\hline $\begin{array}{l}\text { Peripheral Vestibular } \\
\text { Hypofunction }\end{array}$ & $\begin{array}{l}\text { Observable delay of the vestibulo-occular reflex (VOR) } \\
\text { during the bilateral Head Thrust Maneuver and loss of } 2+ \\
\text { lines of visual acuity during the Dynamic Visual Acuity } \\
\text { test (DVA) }\end{array}$ & $\begin{array}{l}\text { Balance exercises in sitting and standing positions that } \\
\text { include gaze stabilization, kinesthetic and propriceptive } \\
\text { retraining. }\end{array}$ \\
\hline $\begin{array}{l}\text { Peripheral motion } \\
\text { hypersensitivity }\end{array}$ & $\begin{array}{l}\text { Motion Sensitivity Quotient (MSQ) elicits symptoms } \\
\text { during or immediately after assuming provocative posi- } \\
\text { tions, and establish symptom onset noting latency and } \\
\text { duration }\end{array}$ & $\begin{array}{l}\text { Habituation exercises that reproduce the provocative mo- } \\
\text { tion, seated and standing balance exercises with gaze } \\
\text { stabilization, kinesthetic and proprioceptive retraining }\end{array}$ \\
\hline $\begin{array}{l}\text { Benign paroxysmal po- } \\
\text { sitional vertigo }\end{array}$ & $\begin{array}{l}\text { Motion Sensitivity Quotient (MSQ) produces symptoms } \\
30 \text { seconds or more after assuming provocative } \\
\text { positions. }\end{array}$ & Canalith repositioning maneuvers \\
\hline
\end{tabular}

